\title{
Estimation of Rock Particle Size Distribution Using Wavelet Decomposition and Morphological Operations
}

\author{
Anthony Amankwah and Chris Aldrich
}

\begin{abstract}
Estimation of the amount of fines in crushed rock from images using standard segmentation algorithms is difficult, owing to the heterogeneity and partial obscuring of the objects to be identified. In this paper, we present an image classification system to estimate the proportion of fines in aggregates of rock particles based on two-level wavelet decomposition and morphological operations. Morphological opening and closing filtering performed on rock images with structuring elements of increasing size also enhanced the class separability of the images of rock particulates. Experimental results showed that the performance of the image classification approach can be superior to standard methods.
\end{abstract}

Index Terms-Image-classification system, morphological operations, rock particulate size, wavelet.

\section{INTRODUCTION}

Information on the size distribution of particles is important for the efficient operation of process plants in the mining industry. In the coal industry, fine material size can lead to channel flow of gases through solid burdens in reactors and significant operational inefficiencies. In the mining industry, particle size distributions are typically measured by using sieves, cyclones and sedimentation techniques. These methods are not only time consuming and expensive, but also do not provide information that can be used for online control or process improvement. Image analysis is becoming increasingly attractive as the cost of computation continuous to decrease, and with it, the implementation of machine vision systems for rock size analysis is becoming more feasible [1]. Some of the current commercial applications available for this purpose, include Split and Wipfrag [2]-[4].

To analyze rock particles, four major processes need to be accomplished in a machine vision system. These are image capture and enhancement, segmentation, feature extraction and the application of domain information to recognize objects. The most difficult step in the measurement of rock particle sizes on conveyer belts is segmentation. Thus, in this paper we propose an image classification methodology for rock particulate size estimation that do not depend on image segmentation. We use wavelet transforms, owing to their

Manuscript received October 30, 2012; revised December 10, 2012.

Anthony Amankwah was with the Department of Process Engineering, University of Stellenbosch, South Africa. He is now with the School Computer Science, University of Witwatersrand, Private bag 3, Wits 2050, Johannesburg, South Africa (e-mail: Anthony.amankwah@wits.ac.za).

Chris Aldrich is with the Western Australian School of Mines, Curtin University of Technology, Perth, Australia (e-mail: chris.aldrich@curtin.edu.au). multi-resolution representation of images and its efficiency with regard to textural feature extraction. Morphological operations using increasing structuring element sizes is also used to extract features that differentiate the rock images with the varying amounts of fines (rock particles with sizes below $6 \mathrm{~mm}$ in this case). The amounts of fines in test rock images are determined by averaging the votes of the $\mathrm{k}$ closest training data points in the feature space.

\section{WAVELET TRANSFORM}

The wavelet transform is a popular tool for texture analysis, as it provides useful information about the spatial and frequency features of the images. In contrast, Fourier transforms for example, show only the frequency characteristics of the pattern [5]. Since the correlation of identical patterns at different scales can be low, multiresolution methods are better able to deal with the problem of scale.

In two dimensions, a two-dimensional scaling function $\Phi(x, y)$, and three two-dimensional wavelets $\Psi^{H}(x, y), \Psi^{V}(x, y)$ and $\Psi^{D}(x, y)$ are required Each is the product of a one-dimensional scaling function $\Phi$ and corresponding wavelet $\Psi$. Excluding products that produce one-dimensional results, like $\Phi(x) \Psi(x)$, the four remaining products produce the separable scaling function $\Phi(x)=\Phi(x) \Phi(y)$ and separable directional wavelets $\Psi^{H}(x, y)=\Psi(x) \Phi(y), \quad \Psi^{V}(x, y)=\Phi(x) \Psi(y)$, $\Psi^{D}(x, y)=\Psi(x) \Psi(y)$.

Given separable two-dimensional scaling and wavelet functions, the scaled and translated bases are as follows:

$$
\begin{gathered}
\Phi_{j, m, n}(x, y)=2^{j / 2} \Phi\left(2^{j} x-m, 2^{j} y-n\right) \\
\Psi^{i}{ }_{j, m, n}(x, y)=2^{j / 2} \Psi^{i}\left(2^{j} x-m, 2^{j} y-n\right) \\
i=\{H, V, D\}
\end{gathered}
$$

where index $i$ identifies the directional wavelets. The discrete wavelet transform of image, $f(x, y)$ of size $M \times N$, is

$$
\begin{aligned}
& W_{\Phi}\left(j_{0}, m, n\right)=\frac{1}{\sqrt{M N}} \sum_{x=0}^{M-1} \sum_{y=0}^{N-1} f(x, y) \Phi_{j_{0}, m, n}(x, y) \\
& W_{\Psi}^{i}\left(j_{0}, m, n\right)=\frac{1}{\sqrt{M N}} \sum_{x=0}^{M-1} \sum_{y=0}^{N-1} f(x, y) \Psi_{j_{0}, m, n}^{i}(x, y) \\
& i=\{H, V, D\},
\end{aligned}
$$


where $j_{0}$ is an arbitrary starting scale. The $W_{\Phi}\left(j_{0}, m, n\right)$ coefficients define an approximation of $f(x, y)$ at scale $j_{0}$.

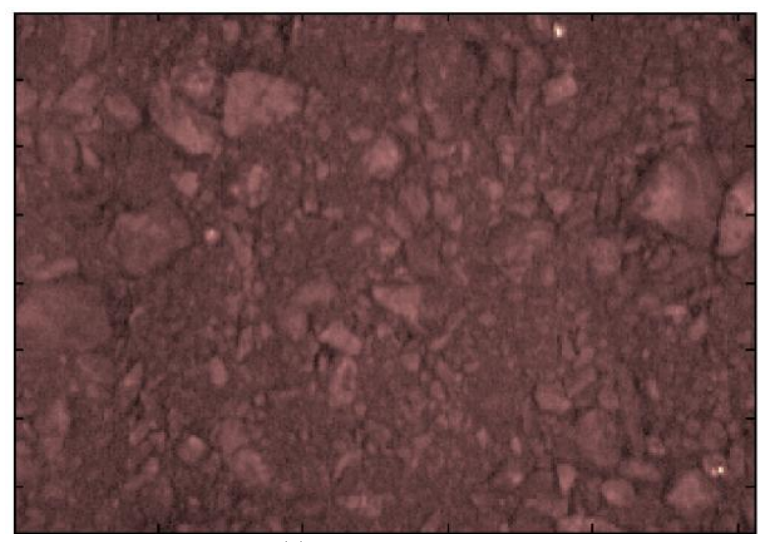

(a)

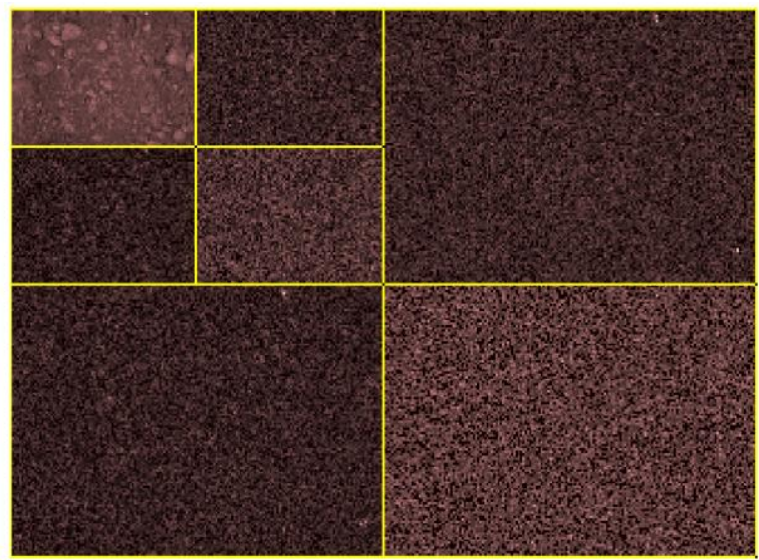

(b)

Fig. 1. Two-level wavelet transform of rock image.

The $W_{\Psi}^{i}\left(j_{0}, m, n\right)$ coefficients add horizontal, vertical, and diagonal details for scales $j \geq j_{0}$. Normally, $j_{0}=0$ and $\quad N=M=2^{J}$ so that $j=0,1,2, \ldots, J-1$ and $m, n=0,1,2, \ldots, 2^{j-1}$.

The equations show that the wavelet transform is a measure of the similarity of the basis functions and the images. Therefore, the wavelet coefficients refer to the closeness of the image to the wavelet at the current scale. In this work, Debauchies wavelet basis functions were used [6]. Fig. 1(b) shows the two-level wavelet transform of the image of rock particles in Fig. 1 (a).

\section{MorphologicAl OPERATIONS}

Mathematical morphology is the analytical theory of spatial structures [6] and is useful for the analysis of shapes of objects of interest in images.

\section{A. Morphological Opening}

Morphological opening of an image $f(x, y)$ is the erosion of $f(x, y)$ followed by dilation with the reflected structuring element $S E$ :

$$
f \circ S E=(f \Theta S E) \oplus S E,
$$

where $\Theta$ and $\oplus$ denote erosion and dilation respectively.
The structural element must be set according to the objects of interest and since rock particles normally have approximately ellipsoidal shapes, the structuring element used in this work was an ellipse.

\section{B. Morphological Closing}

Morphological closing is used to enhance the original shape of the objects of interest, based on erosion of the dilated image.

$$
f \bullet B=(f \oplus S E) \Theta S E
$$

Fig. 2 shows examples of closings openings with different sizes of the structuring element.

\section{FEATURE EXTRACTION}

Feature extraction is an essential stage in texture classification and is critical to the success of texture classification systems. The main difference between many texture classification systems is the feature extraction method. In this study, the values of the calculated wavelet coefficients and the pixel values of the morphologically filtered images were the essential features that were used to determine the proportion of fines in samples of rock aggregates. To reduce computational cost, the following features were used, viz. the energy of the images after morphological processing, as well as the mean, variance, entropy and contrast of the wavelet coefficients of selected subbands calculated from the co-occurrence matrix of the particular subbands [7]-[8]. Table I shows the features used classify the amount of fines in rock images

TABLE I: FEATURES COMPUTED FROM GRAY LEVEL CO-OCCURRENCE MATRIX $F(X, Y)$

\begin{tabular}{ll} 
Features & $\sum_{x} \sum_{y}|f(x, y)|^{2}$ \\
\hline Energy & $\sum_{x} \sum_{y} f(x, y)$ \\
Entropy & $\sum_{x} \sum_{y} f(x, y) \log (f(x, y)$ \\
& \\
Variance & $\sum^{\prime} \mid f(x, y)-$ mean $\left.\right|^{2}$
\end{tabular}

\section{IMAGE ClassificAtion SYSTEM}

Our proposed classification based size estimation system uses the k-Nearest Neighbor to estimate the amount of fines in rock samples. The amount of fines is defined as follows:

$$
S \hat{D}(V)=\frac{\sum_{S D_{i}(V) \in N_{k}(V)} S D_{i}(V)}{k}
$$

where $S D_{i}(V)$ and $N_{k}(V)$ are the k-closest training features and their neighborhoods respectively. We calculate the Euclidean 
distances of the query image and all the training set images and find the k-closest neighbors. The average of the k-closet neighbors in the feature space is the estimate of amount of fines in rock sample

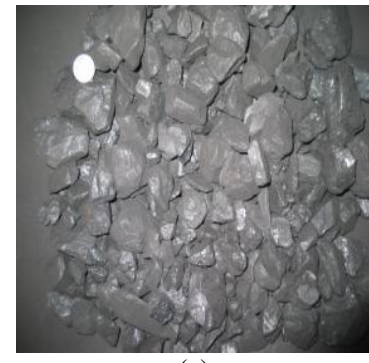

(a)

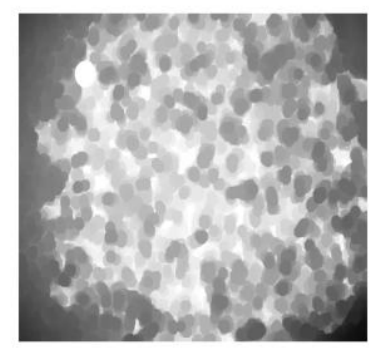

(b)

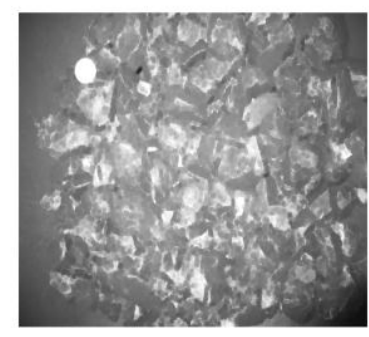

(c)

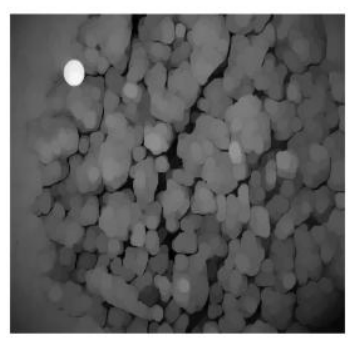

(d)

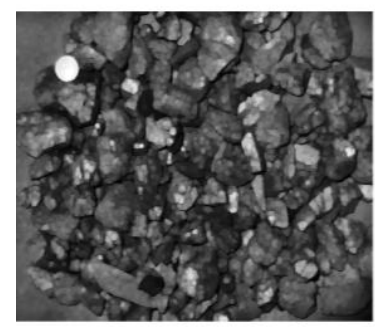

(e)

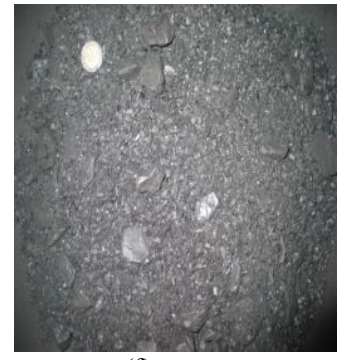

(f)
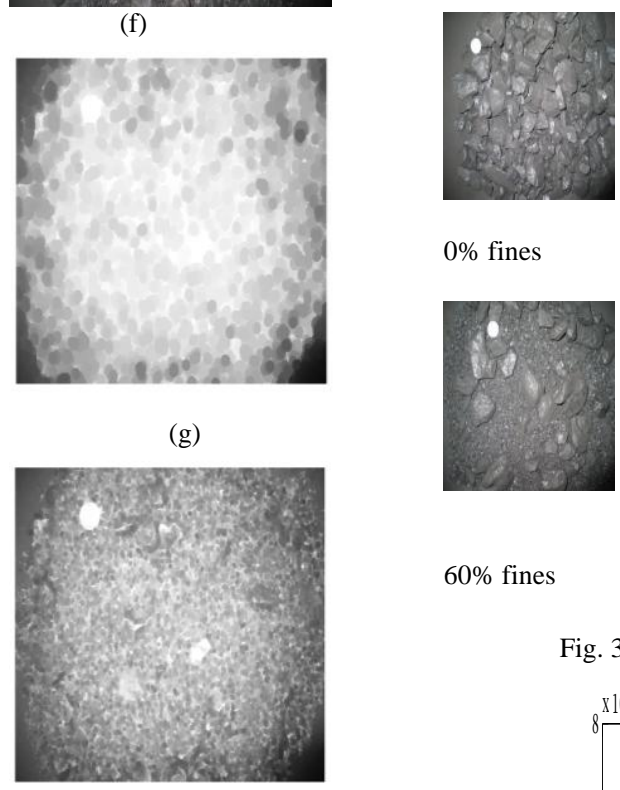

(h)

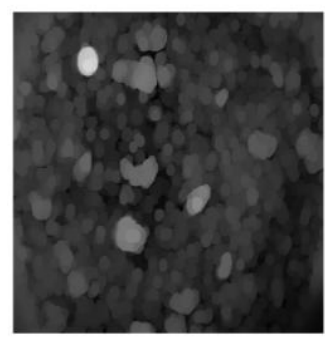

(i)

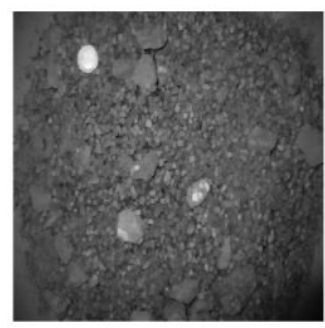

(j)
Fig. 2. (a) Original rock image with $0 \%$ fines (b) Morphological closing of image in (a) using structuring element (SE) of size 30 (c) Morphological closing of image in (a) using a SE of size 10. (d) Morphological opening of image in (a) using a SE of size 30 (e) Morphological opening of image in (a) using a SE of size 10 (f) Rock image with $80 \%$ fines (g) Morphological closing of image in (f) using SE of size 30 (h) Morphological closing of image in (f) using SE of size 10 (i) Morphological opening of image in (f) using SE of size 30 (j) Morphological opening of image in (f) using SE of size 10 . fractions.
$0 \%$ fines

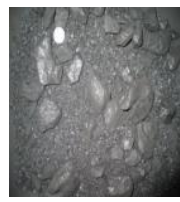

\section{EXPERIMENTS AND RESULTS}

In order to compare our proposed method and standard methods, our image classification method and the commercial Split software were tested on prepared coal samples with different amounts of fines. Coal samples were sieved on a pilot plant in to $-6 \mathrm{~mm}$ (fines) and $+6 \mathrm{~mm}$ (coarse)

Seven different blends were created consisting of $0 \%, 20 \%$, $40 \%, 50 \%, 60 \% 80 \%$ and $100 \%$ fines as shown in Fig. 3. Ten samples of each blend were prepared, of which five samples of each blend were used as training data. The rest of the images were used as test data.
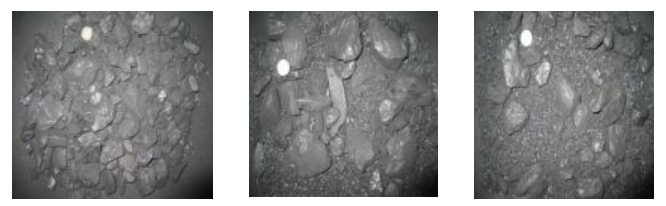

$20 \%$ fines

$40 \%$ fines

$50 \%$ fines
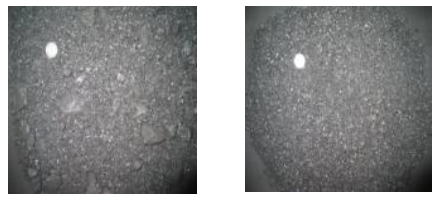

$60 \%$ fines

$80 \%$ fines

$100 \%$ fines

Fig. 3. Rock image showing different amounts of fines.

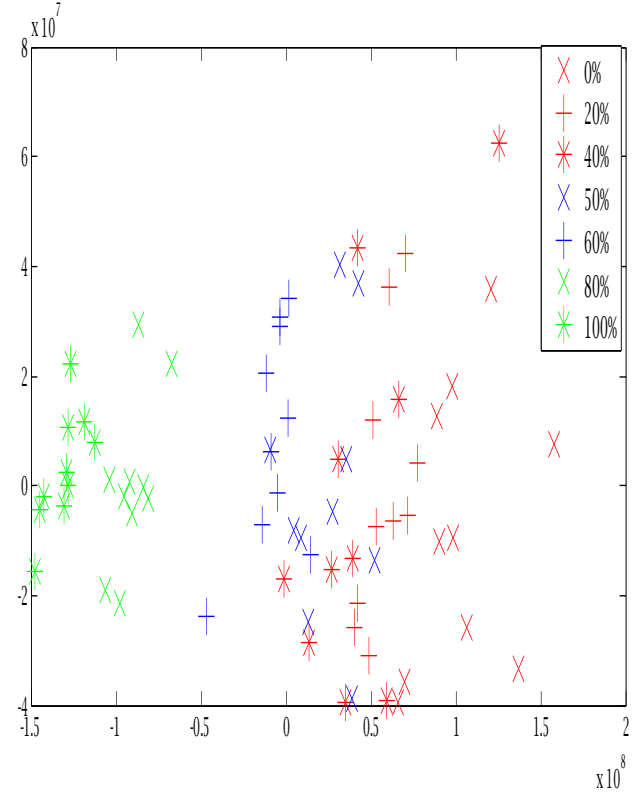

Fig. 4. Principal component score plots of grades of fines in two dimensions.

Principal component analysis (PCA) was used for visualization of the selected features by projecting them onto a two-dimensional plane, as shown in Fig. 4. In this figure, 70 different samples of the seven blends of rock imagesare shown. As indicted by Fig. 4, there were no sharp differences between the blends with $40 \%$ fines and those with $50 \%$ fines. This is partly due to the similarity of the images in the range. Fig. 5 shows the results of the estimation of fines by use of the image classification system discussed above and Split software. Estimates are compared with the ground truth on 35 rock image samples. The average sum of absolute difference 
between the ground truth, the proposed method and Split software are 6.1 and 23.3 respectively.

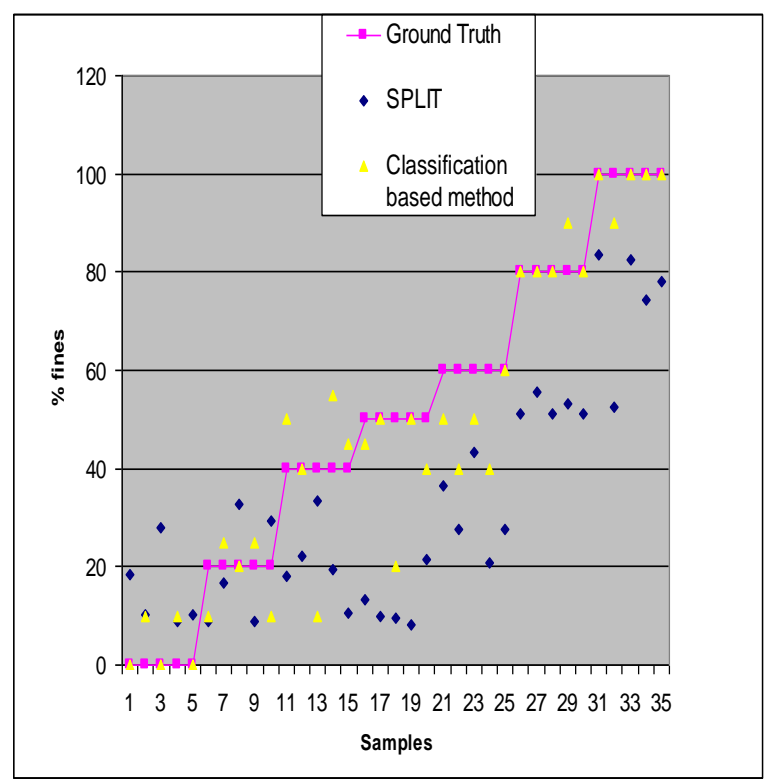

Fig. 5. Results of estimation of amount of fines by algorithms.

\section{CONCLUSION}

In this work, we have presented a classification based method for estimating the amount of fines in rock image. The method uses the wavelet transform and morphological operations to extract features. Experimental results show that the performance of image classification system can be better than standard methods used, as represented by the Split algorithm. Moreover, the approach is general and readily adaptable to similar particle size estimation problems.

\section{REFERENCES}

[1] G. C. Hunter, C. McDermott, N. J. Miles, and A. Singh, "A review of image analysis techniques for measuring blast fragmentation," Mining Science and Technology, vol. 11, no. 1, pp. 19-36, 1990
[2] J. Kemeny, A. Devgan, R. Hagaman, and X. Wu, "Analysis of rock fragmentation using digital image processing,", Geotech. Eng., vol. 199, no. 7, pp. 1144-1160, 1993

[3] Software Service. (March 2009). [Online]. Available: http://www.spliteng.com/ Accessed 2

[4] N. H. Maerz, T. C. Palangio, and J. A. Franklin, "Wipfrag image based granulometric system," Meas. Blast Fragment, pp. 91-99, 1996.

[5] S. G. Mallat, "A theory for multi-resolution signal decomposition: The wavelet representation," IEEE Transactions on Pattern Analysis and Machine Intelligence, vol. 11, no. 7, pp. 647-693, 1989.

[6] Serra, Mathematical Morphology, Academic Press, London, UK, vol 11982.

[7] I. Daubechies, "Orthonormal bases of compactly supported wavelets," Communications on Pure and Applied Mathematics, vol. 41, pp. 909-996.

[8] R. M. Haralick, K. Shanmugan, and I. Dinstein, "Textural Features for Image Classification," IEEE Transactions on Systems, Man, and Cybernetics, vol. SMC-3, 1973, pp. 610-621.

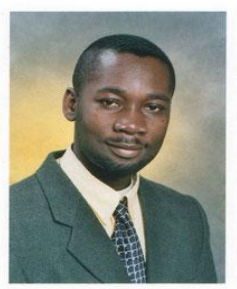

Anthony Amankwah received his B.Sc. degree in Metallurgical Engineering from the Kwame Nkrumah University of Science and Technology, Kumasi, Ghana in 1996 and B.Sc., M.Sc. degrees in Electrical Engineering and Computer Science from the University of Duisburg-Essen, Duisburg, Germany in 2003. He the received a Ph.D. degree in Electrical Engineering and Computer Science from the University of Siegen, Siegen, Germany.

$\mathrm{He}$ is currently a Lecturer the University of Witwatersrand, Johannesburg, South Africa. During the period 2000-2003, Dr. Amankwah held a KAAD scholarship from the Catholic Academic Exchange Service, Bonn, Germany. His research interest include machine vision, image processing, robotics, and intelligent systems.

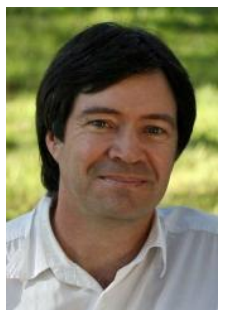

Chris Aldrich is a fellow of the South African Academic of Engineering and a professor in the Department of Metallurgical and Minerals Engineering at the Western Australian School of Mines at Curtin University in Australia His research interests include machine learning, data mining and advanced process modeling and control systems in the chemical and mineral processing industries. 